\title{
TRANSIENT ABNORMAL MYELOPOIESIS IN A NEONATE
}

Ketan P. Mallya, Deepak Nayak M., Sushma V. Belurkar, Chethan Manohar

1. Speciality Medical Officer, Department of Pathology, Melaka Manipal Medical College, Manipal, Manipal University

2. Assistant Professor, Department of Pathology, Melaka Manipal Medical College, Manipal, Manipal University

3. Associate Professor, Department of Pathology, Kasturba Medical College, Manipal, Manipal University.

4. Professor, Department of Pathology, Kasturba Medical College, Manipal, Manipal University.

\section{CORRESPONDING AUTHOR}

Dr. Deepak Nayak M.

Department of Pathology,

Melaka Manipal Medical College,

Manipal University, Manipal 576104, India.

E-mail: deepzienator@gmail.com

Ph: 00919901920537

ABSTRACT: Transient abnormal myelopoiesis (TAM) is a unique disorder of newborns associated intimately with Down's syndrome, presenting with clinical and morphological features indistinguishable from acute myeloid leukaemia (AML). We report a case in a neonate, presenting with severe perinatal asphyxia and cyanosis; complicated by metabolic acidosis. The hemogram revealed leucocytosis and thrombocytopenia. The peripheral smear showed marked left shift and 55\% circulating myeloblasts. Additional findings included a hepatomegaly and mild dysmorphic features. The child eventually succumbed to pulmonary hemorrhage on day one itself. TAM has to be differentiated from congenital leukemia which portends a poor prognosis.

KEY WORDS: Transient abnormal myelopoiesis, Down's syndrome, neonate

INTRODUCTION: Neonates and infants with Down's syndrome (DS) infrequently develop a condition known as transient abnormal myelopoiesis (TAM). It has been referred to in literature by varied nomenclature such as transient leukemia (TL) or transient myeloproliferative disorder (TMPD).The majority of infants with TAM are asymptomatic, being picked up incidentally on routine hematologic testing. Most cases of TAM are known to resolve over the first few months of life, a small percentage may go on to develop AML within the first few years of life.

CASE REPORT: We present here a male neonate born to a 36 year-old mother (G2 P1 L1) by full term normal vaginal delivery; who had a supervised pregnancy with regular antenatal care. The anomaly scan was normal. The baby did not cry at birth. The neonate weighed $3 \mathrm{~kg}$ at birth and had bradycardia (heart rate $<60 / \mathrm{min}$ ). The Apgar score was not known .The respiratory efforts were poor and cyanosis was recorded at birth. On further examination, the baby had mild dysmorphic features with a palpable liver ( $4 \mathrm{~cm}$ beneath the right costal margin). The child had generalized edema and poor CNS reflexes.

The child was placed on High Frequency Oscillatory mechanical ventilation with inotropic support. 
LABORATORY FINDINGS: The coulter hemogram revealed anaemia and thrombocytopenia ( $\mathrm{Hb}-11 \mathrm{~g} \%$ and platelet count $\left.-<100 \times 10^{3} / \mathrm{cu} . \mathrm{mm}\right)$. More significantly, a marked leucocytosis was noted with a total leucocyte count of $174 \times 10^{3} / \mathrm{cu}$.mm (Fig 1).

The peripheral smear confirmed the above findings, additionally showing spherocytosis, anisopoikilocytosis and 77nRBCs/100WBCs. The differential count showed marked left shift up to blasts. Myeloblasts accounted for $55 \%$ of the leucocytes, showing moderate to large sized cells with high N/C ratio, dispersed chromatin; few showing a single inconspicuous nucleolus(Fig 2)

In the light of above morphological features and the available clinical data, a provisional diagnosis of transient abnormal myelopoiesis (TAM) was given on the peripheral smear. A request was made to send the peripheral venous blood sample to flow cytometry for immunophenotyping of the blasts. We also advised a bone marrow study if it was clinically indicated.

The neonatal asphyxia was compounded by a severe metabolic acidosis and elevated liver enzymes; and the child succumbed to pulmonary hemorrhage within few hours of birth, on day one, despite adequate resuscitative measures.

DISCUSSION: Transient abnormal myelopoiesis (TAM) is the clonal proliferation of myeloid blast cells which affects an estimated $5-10 \%$ of newborns with Down'ssyndrome. ${ }^{1}$ It is also uncommonly described in phenotypically normal neonates with trisomy 21 mosaicism. ${ }^{2}$ The majority cases of TAM are asymptomatic at presentation and picked up incidentally on routine hematological testing. Most cases of TAM resolve with spontaneous remission over the first few months of life, but 13-33\%may go on to develop AML within the first four years of life. ${ }^{3}$

Only a handful of cases presenting within few hours of birth have been described. The typical clinical picture of a patient requiring treatment is one of an unwell neonate or an infant on intensive care with an oxygen or inotrope requirement or serious bleed. ${ }^{3}$ These clinical features were consistent with our case. Systemic examination findings may include hepatomegaly or even hepatosplenomegaly. 2,3. Rarely clinical complications include cardiopulmonary failure, hyperviscosity, splenic necrosis and progressive hepatic fibrosis. ${ }^{4}$ The present case concurred partially with these complications; showing cardiac and respiratory adversities.

Neonates with Down's syndrome are unique in having a predisposition to transient abnormal myelopoiesis. One of the reasons hypothesized is that trisomy 21 affects fetal liver hematopoiesis causing an increase in the megakaryocyte- erythroid progenitor frequency with common myeloid progenitors and a reduction in the granulocyte-monocyte progenitors. ${ }^{5}$ Increased clonogenicity affecting megakaryocyte-erythroid progenitors, increased granulocytemonocytes and colony-forming unit-granulocytes has also been found. ${ }^{6}$

GATA-1 mutations, a transcription factor mutation integral to the normal development of erythroid, megakaryocytic and basophilic cell lines, have been associated with TAM and AML in Down's syndrome, as elucidated by studies done by Tunstall- Pedoe and colleagues. ${ }^{7}$ As such, TAM and myeloid leukaemia of Down's syndrome provides an excellent model for leukaemogenesis. ${ }^{8}$

TAM is seen as an increase in the number of blasts on peripheral blood film. Thrombocytopenia is the most common presentation; with anemia being less frequently encountered.2. This hematological finding was noted in our case as well. There may be marked leucocytosis with fewer blasts in the marrow than peripheral blood.2,3 
The peripheral blood and bone marrow blasts in TAM have basophilic cytoplasm with cytoplasmic blebbing, suggestive of megakaryoblasts. ${ }^{2}$ Blasts in TAM display a characteristic immunophenotype. ${ }^{9}$ In most cases the blasts are positive for CD34, CD56, CD117, CD13, CD33, CD7, CD4 (dim), CD4, CD42, TPO-R, CD36, CD61 and CD71.The blasts are negative for MPO, CD15, CD14 and glycophorin A. Antibodies to CD41 and CD61 are considered useful in identifying cells with megakaryocytic lineage in immunologic preparations. ${ }^{2}$ Due to the early death of the neonate in our case, flow cytometry could not be performed.

Factors associated with early death in TAM include a high white cell count, increased liver enzymes, failure to normalize the blood count, ascites, preterm delivery, bleeding diatheses and failure of spontaneous remission. ${ }^{1}$ Three of these variables were seen in our case as well, probably accounting for the early neonatal death. Interestingly, TAM can occur in-utero and be a cause of foetal death. ${ }^{3}$

Morphologically distinguishing TAM from congenital acute leukaemia is a diagnostic challenge for a hematopathologist; especially in smaller centers where ancillary studies may not be available. The difference in survival rates (being in favour of TAM) also merits a morphological differentiation. Also, with the incidence of overt acute leukaemia being markedly increased in infants with Down's syndrome, the distinction between TAM and acute leukaemia is imperative. The characteristics common to both TAM and congenital acute leukaemias include a clonal nature, as demonstrated by molecular studies and an association with Down's syndrome. Trisomy 21 is the recurrent chromosomal anomaly in both conditions. However, TAM may have cryptic inversions of chromosome 21 as opposed to interstitial deletions of $21 \mathrm{q}$ in acute leukaemias. ${ }^{10,11,12}$.The differentiating features particular to acute leukaemia include ${ }^{10}$ presentations after 6 months of age and variable WBC counts with striking dyspoiesis. The circulating blasts in acute leukaemia are more phenotypically uniform; as opposed to a more heterogeneous blast population in TAM.13,14,15 The bone marrow study usually reveals a diffuse replacement of the marrow by blasts in acute leukaemia whereas TAM may have a lower percentage of blasts than in peripheral blood. The spectrum of blasts in TAM ranges from myeloid (typically lacking Auer rods), megakaryoblasts (being more abundant) and erythroblasts. Acute leukaemia on the other hand comprises of megakaryoblastic (in children $<3$ years) or lymphoblastic in older children ( $>3$ years). While TAM has a good prognosis, dictated by a spontaneous resolution in 2-3 months; acute leukaemia portends a poor prognosis, mandating an aggressive chemotherapy.

CONCLUSION: Transient abnormal myelopoiesis (TAM) is a condition with clonal proliferation of myeloid blast cells in newborns with Down's syndrome. Recognizing this entity is of clinical and academic importance since it carries a better prognosis compared to its more sinister counterpart, congenital leukaemia.

\section{REFERENCES:}

1. Klusmann JH, Creutzig U, Zimmermann M, Dworzak M, Jorch N, Langebrake C, et al. Treatment and prognostic impact of transient leukaemia in neonates with Down's syndrome. Blood. 2008; 111: 2991-2998.

2. Baumann I, Niemeyer CM, Brunning RD, Arber DA, Porwit A. Myeloid proliferations related to Down'ssyndrome. In: WHO Classification of Tumours of Hematopoietic and Lymphoid Tissues. Swedlow SH et al (editors).IARC, Lyon 2008:142-144. 
3. Payne E,Salamt A, Connor P. Transient abnormal myelopoiesis in an infant with Down's syndrome. Libyan J Med. 2010, 5: 4635 - DOI: 10.3402/ljm.v5i0.4635.

4. Dixon N. Kishnani PS, Zimmerman S. Clinical manifestations of hematologic and oncologic disorders in patients with Down's syndrome. Am J Med Genet C Semin Med Genet.2006; 142:149-157.

5. Webb D, Roberts I, Vyas P. Haematology of Down's syndrome. Arch Dis Child Fetal Neonatal. Ed. 2007; 92:503-507.

6. Tunstall-Pedoe O, Roy A, Karadimitris A, de la Fuente J, Fisk NM, Bennett P, et al. Abnormalities in the myeloid progenitor compartment in Down's syndrome fetal liver precede acquisition of GATA-1 mutations. Blood. 2008; 112: 4507-4511.

7. Ahmed M, Sternberg A, Hall G, Thomas A, Smith 0, O'Marcaigh A, et al. Natural history of GATA-1 mutations in Down syndrome. Blood. 2004; 103: 2480-2409.

8. Vyas P, Roberts I. Down's myeloid disorders: a paradigm for childhood preleukaemia and leukaemia and insights into normal megakaryopoiesis. Early Hum Dev. 2006; 82: 767-773.

9. Langebrake C, Creutzig U, Reinhardt T. Immunophenotype of Down's syndrome acute myeloid leukaemia and transient myeloproliferative disease differs significantly from other diseases with morphologically identical or similar blasts. Klin Padiatr.2005; 217:126-134.

10. Foucar K, Brynes R, Head D, Sever C. Special Considerations for Bone Marrow in Children. Foucar K, editor. Bone Marrow Pathology. 2nd ed. Chicago: ASCP Press; 2001.p.587-607.

11. Kempski HM, Chessels JM, Reeves BR. Deletions of chromosome 21 restricted to leukaemic cells of children with Down's syndrome and leukaemia. Leukemia.1997; 11:1973-1977.

12. Kempski HM, Craze JL, Chessels JM, Reeves BR. Cryptic deletions and inversions of chromosome 21 in a phenotypically normal infant with transient abnormal myelopoiesis; a molecular cytogenetic study. Br J Hematol.1998; 103: 473-479.

13. Avet-Loiseau H, Mechinaud F, Harousseau JL. Clonal hematologic disorders in Down'ssyndrome; a review. J Pediatr Hematol Oncol.1995; 17:19-24.

14. Litz CE, Davies S, Brunning RD, et al. Acute leukemia and the transient myeloproliferative disorder associated with Down's syndrome: morphologic, immunophenotypic and cytogenetic manifestations. Leukemia. 1995; 9:1432-1439.

15. Foucar K, Friedman K, Llewellyn A, et al. Prenatal diagnosis of transient myeloproliferative disorder via percutaneous umbilical cord blood sampling: report of two cases in fetuses affected by Down's syndrome. Am J Clin Pathol. 1992; 97:584-590. 


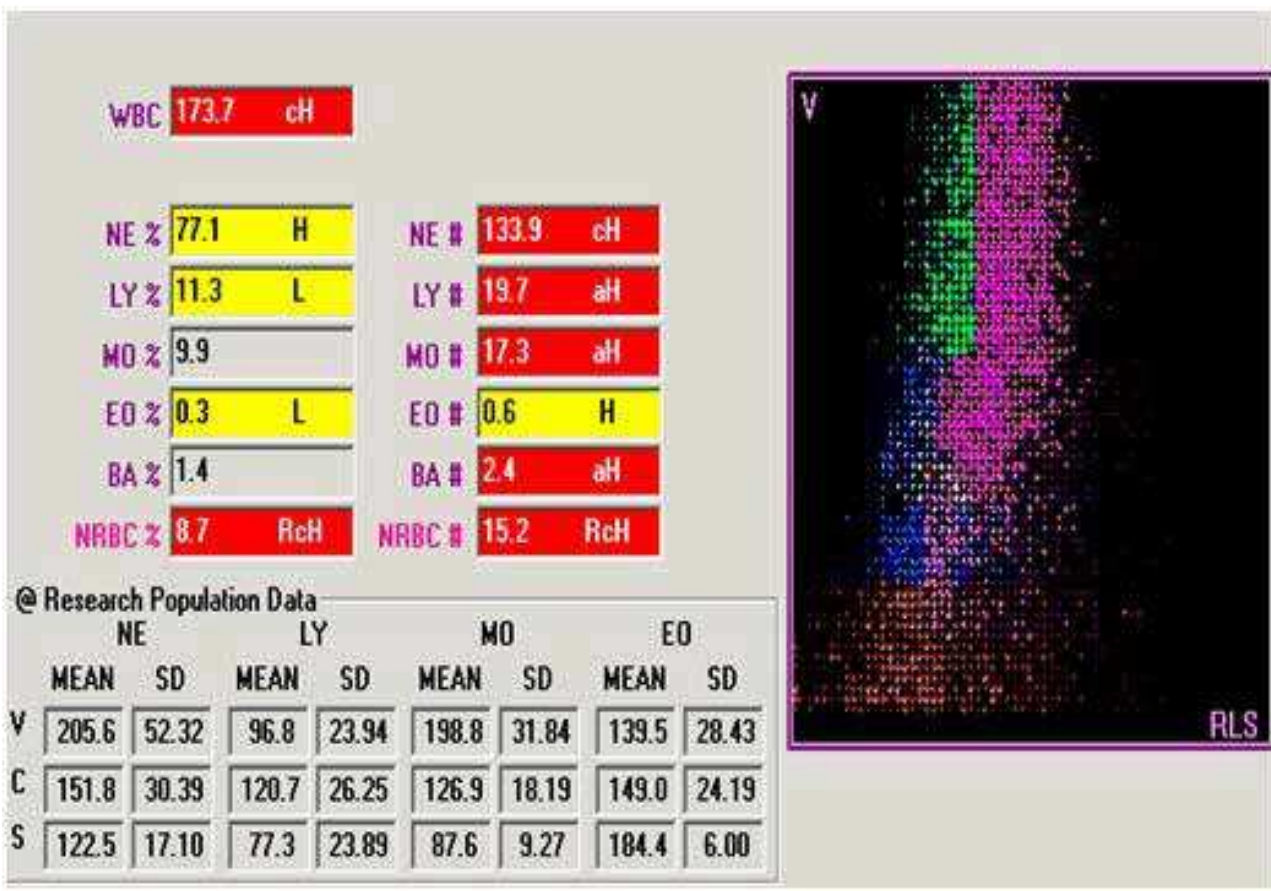

Fig 1: LH750 Analyzer (Beckman Coulter) hemogram revealing leucocytosis. The five part differential scatter plot showing a population of immature cells

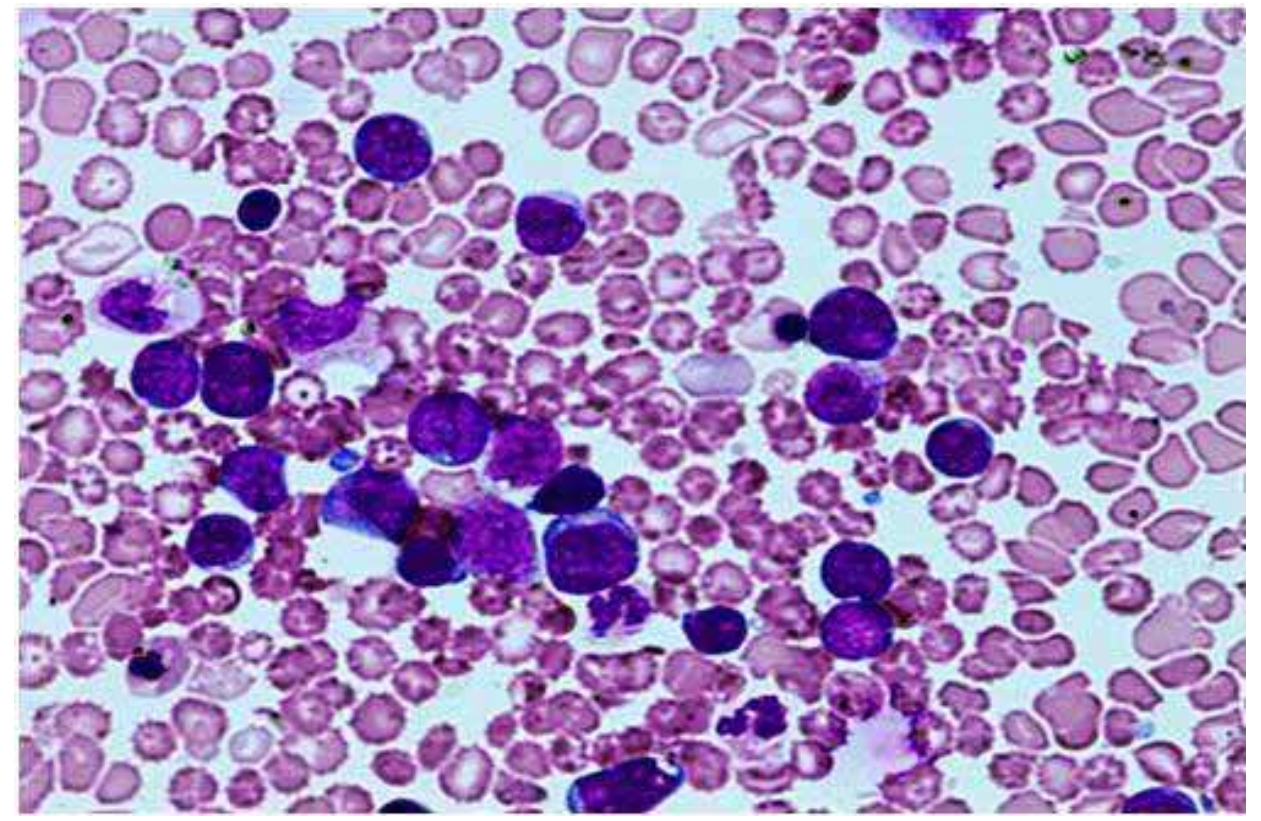

Fig 2: Peripheral smear showing marked left shift with increase in myeloblasts (Leishman x 200) 